\title{
Medicamentos Biotecnológicos: Requisitos Exigidos para el Desarrollo y Aprobación de Biosimilares
}

\author{
Begoña Calvo* y Leyre Zúñiga \\ Universidad del País Vasco / EHU, Facultad de Farmacia, Depto. de Farmacia y Tecnología \\ Farmacéutica, Po Universidad, 7. 01006 Vitoria-España (e-mail: b.calvo@ehu.es) \\ * Autor a quien debe ser dirigida la correspondencia
}

Recibido Dic. 24, 2009; Aceptado Feb. 18, 2010; Versión Final recibida Abr. 19, 2010

\begin{abstract}
Resumen
En este artículo se revisan las directrices europeas de comparabilidad que establecen la metodología para la determinación de biosimilitud entre los medicamentos biosimilares (follow-on biologics en USA) y el medicamento biológico de referencia. Los biosimilares son medicamentos biológicos parecidos pero no idénticos al medicamento original y pueden ser fabricados por cualquier fabricante al finalizar el periodo de patente de los medicamentos biotecnológicos. En el articulo se detallan las directrices de la Agencia Europea del Medicamento (EMA) y de la Conferencia Internacional de Armonización ( $\mathrm{ICH}$ ) a tener en cuenta en el desarrollo y aprobación de estos medicamentos. Se demuestra que los medicamentos biosimilares no pueden considerarse medicamentos genéricos, siendo necesario realizar una serie de ensayos adicionales previos a la obtención de la autorización de comercialización.
\end{abstract}

Palabras clave: biosimilares, medicamentos biotecnológicos, regulación de medicamentos, medicamentos genéricos

\section{Biological Medicinal Products: Requirements for the Development and Approval of Biosimilars}

\begin{abstract}
This article reviews the European guidelines on drugs comparability that establish the methodology for verifying biosimilarity between the so-called biosimilar drugs and the reference biological medicinal product. Biosimilars are biological medicines similar but not identical to the original drugs and can be manufactured by any laboratory after the expiration of biotech drugs patent. The guidelines of the European Medicines Agency (EMA) and the International Conference on Harmonization (ICH) that must be considered in the development and approval of these drugs also are reviewed. It is shown that biosimilars cannot be considered as generic drugs, being necessary to conduct additional assays prior to obtain marketing authorization.
\end{abstract}

Keywords : biosimilars, biotech drugs, drugs regulation, generic drugs 


\section{INTRODUCCIÓN}

A comienzos del año 2004, la protección de las patentes de la primera generación de medicamentos biotecnológicos comenzaba a expirar, dejando las puertas abiertas a los fabricantes de biosimilares (follow-on biologics en Estados Unidos) y obligando a las autoridades sanitarias a revisar los procedimientos actuales de registro de medicamentos puesto que el origen biológico de los biosimilares les confiere una especificidad que la normativa actual sobre medicamentos genéricos no es capaz de abarcar.

Un medicamento biosimilar es un medicamento parecido a un medicamento biológico que ya ha sido autorizado (el "medicamento biológico de referencia"). La sustancia activa de un medicamento biosimilar es parecida a la del medicamento biológico de referencia. Los medicamentos biosimilares y de referencia se utilizan generalmente a la misma dosis para tratar la misma enfermedad. Teniendo en cuenta que ambos medicamentos son parecidos pero no idénticos, la decisión de tratar a un paciente con el medicamento de referencia o el biosimilar debe tomarse en base a la opinión de un profesional sanitario cualificado. El nombre, apariencia y empaquetado de un medicamento biosimilar difieren de los del medicamento biológico de referencia (EMEA, 2007).

\section{Definición de medicamento biosimilar}

Las directrices de la Agencia Europea del Medicamento (EMEA) consideran a los biosimilares como entidades totalmente diferentes a los productos originales y destacan la importancia que tiene el proceso de fabricación de los biofármacos (Crommelin et al, 2005).

Dentro de las diferencias encontradas entre los biosimilares y los medicamentos de referencia correspondientes podemos citar las siguientes (Schellekens y Moors, 2010):

- Patrón de glicosilación diferente: Abseamed, Binocrit, Epoetina alfa Hexal, Retacrit y Silapo.

- Células huésped: Valtropin.

- Composición de la formulación: Retacrit, Silapo, Biograstim, Ratiograstim,Tevagrastim Zarzio y Filgrastim Hexal.

- Contenido en impurezas: Zarzio, Filgrastim Hexal, Abseamed, Binocrit, Epoetina alfa Hexal.

En Octubre de 2005 la EMEA reconoció la complejidad de los biofármacos al publicar un documento que abarca todo el tema (EMEA, 2005), declarando que los biosimilares no son genéricos y que pueden ser objeto de rigurosos ensayos antes de obtener la autorización de comercialización. El resto de directrices generales y específicas de producto para medicamentos biosimilares emitidas por la EMEA se encuentran bajo el amparo de esta Directriz (Zúñiga y Calvo, 2009a). La complejidad de los biofármacos se refleja en los documentos de conceptualización específicos por tipo de biofármaco publicados por esta Agencia.

En Estados Unidos, la aprobación del proyecto de reforma sanitaria por el Congreso en marzo de 2010 ha supuesto el inicio de la regulación legal de los medicamentos biosimilares en este país (HR 3590, 2010). Otros paises también están desarrollando acciones para regular la aprobación de biosimilares. En Australia, por ejemplo, se han adoptado directamente las directrices de la EMEA y en Japón la regulación legal también se ha basado en las directrices establecidas por la EMEA. Por otra parte, en algunos paises como India, China y algunos paises de Latino América, se han autorizado copias de productos biofarmacéuticos, que no en todos los casos cumplen los estándares de calidad, seguridad y eficacia establecidas en las directrices de la EMEA (Kresse, 2009).

\section{DIRECTRIZ DE MEDICAMENTOS BIOLÓGICOS SIMILARES: CHMPI437/04}

Un laboratorio farmacéutico puede elegir desarrollar un nuevo producto biológico como "similar" (EMEA, 2005) a un medicamento de referencia (medicamento innovador) con autorización de comercialización en la UE en base a un dossier de registro completo según lo previsto en la Directiva 2001/83/CE (CE, 2001) y enmiendas posteriores. En esta directiva se establecen los requisitos que 
deben cumplir las solicitudes de autorización de comercialización basadas en la demostración de la naturaleza similar de los dos medicamentos. Para llevar a cabo su desarrollo se requieren estudios comparativos que demuestren la naturaleza similar en lo que se refiere a calidad, seguridad y eficacia entre el nuevo medicamento biológico y el medicamento de referencia ya autorizado en la Unión Europea, son los conocidos como "estudios de comparabilidad" (Zúñiga y Calvo, 2010a) .

Los avances y las limitaciones de los métodos y técnicas disponibles hasta la fecha para la caracterización completa de tales medicamentos, han llevado al Comité de Medicamentos de Uso Humano (CHMP) de la EMEA a desarrollar guías específicas sobre la calidad, los aspectos noclínicos y clínicos que se deben tener en cuenta en el desarrollo de medicamentos biosimilares (Zúñiga y Calvo, 2009a).

Como se ha comentado, los medicamentos biológicos son normalmente más difíciles de caracterizar que los medicamentos de síntesis química debido a la complejidad molecular de estos productos (ADN recombinante, derivados de sangre o plasma, inmunológicos, productos de terapia génica/celular, etc). Además, algunos parámetros como la estructura tridimensional, cantidad de variantes ácido-base o modificaciones post-traducción tales como el perfil de glicosilación pueden alterarse de forma significativa debido a cambios, que inicialmente se consideran "menores" en el proceso de fabricación (Zopf y Vergis, 2002; Covic y Kuhlman, 2007; Roger y Mikhail, 2007). Por lo tanto, el perfil de seguridad-eficacia de estos productos depende en gran medida de la robustez y la monitorización de los aspectos de calidad (Zúñiga y Calvo, 2009b).

\section{Por lo tanto :}

- La aproximación genérica estándar (demostración de bioequivalencia con un medicamento de referencia a través de estudios adecuados de biodisponibilidad) que normalmente es aplicable a los productos obtenidos por síntesis química no es científicamente apropiada para los productos biológicos/biotecnológicos debido a su complejidad. Por tanto, tendrán que aplicarse las guías específicas de "medicamentos biosimilares".

- Resulta más fácil llevar a cabo pruebas comparativas para demostrar similitud con productos altamente purificados, que puedan ser completamente caracterizados (tales como algunos productos obtenidos por biotecnología).

- La posibilidad de aprobación de un medicamento utilizando la normativa de "medicamentos biosimilares" depende del nivel de desarrollo de los procedimientos analíticos, el proceso de fabricación empleado, así como de la experiencia clínica y regulatoria.

- En relación a los datos de calidad, un medicamento biosimilar debe cumplir todos los requisitos del Módulo 3 del Documento Técnico del dossier de registro (Directiva 2001/83/CE) y satisfacer los requisitos técnicos de las monografías de la Farmacopea Europea, además de los requisitos adicionales que se definan en las guías CHMP e ICH (Conferencia Internacional de Armonización) relevantes.

- Los datos utilizados para demostrar seguridad y eficacia de los medicamentos biosimilares deben cumplir los requisitos detallados en el Anexo I de la Directiva 2001/83/CE. Las guías de la EMEA/CHMP contemplan previsiones técnicas generales y específicas de producto y en las situaciones donde no se dispone de guías específicas de producto los laboratorios solicitantes deben demandar consejo a las Autoridades Regulatorias Europeas.

- Hay que tener en cuenta que, por definición, los medicamentos biosimilares no son medicamentos genéricos y que las diferencias entre medicamentos biosimilares de diferentes fabricantes o entre productos de referencia se evidencian a medida que se tiene experiencia en su uso. Por lo tanto, con objeto de reforzar la monitorización de la farmacovigilancia, el medicamento administrado al paciente debe estar claramente identificado, especificando además de su nombre $\mathrm{DCl}$ (denominación común internacional), el nombre comercial y/o fabricante (Kuhlmann y Goldsmith, 2007;Zúñiga y Calvo, 2010b) . 
El medicamento de referencia elegido para realizar los estudios de comparabilidad debe ser un medicamento autorizado en la Unión Europea en base a un expediente completo según lo previsto en la directiva anteriormente citada y enmiendas (ver tabla 3).

Este medicamento de referencia se utilizará a lo largo de todo el programa de comparabilidad para los estudios de calidad, seguridad y eficacia durante el desarrollo del medicamento biosimilar con el fin de obtener datos y conclusiones coherentes. Los datos generados en los estudios de comparabilidad de medicamentos autorizados fuera de la UE solamente pueden aportar información de apoyo.

El principio activo de un medicamento biosimilar debe ser similar, en términos moleculares y biológicos, al principio activo del medicamento de referencia. Por ejemplo, un medicamento que contiene interferón alfa-2a fabricado por el laboratorio $X$ afirmando ser similar a otro medicamento biológico debe compararse con un medicamento de referencia que contenga como principio activo interferón alfa-2a. Por lo tanto, un medicamento que contenga interferón alfa-2b nunca puede considerarse como el producto de referencia.

La forma farmacéutica, la potencia y vía de administración del medicamento biosimilar deben ser las mismas que las del producto de referencia. Cuando la forma farmacéutica, la potencia o la vía de administración no sean iguales, deben especificarse. Cualquier diferencia entre el biosimilar y el medicamento de referencia tendrá que justificarse mediante la realización de estudios apropiados. Siempre que exista cualquier duda al respecto se recomienda consultar con la EMEA (Nowicki, 2007).

Asimismo, se prevé la publicación de anexos adicionales a medida que surja la necesidad y las guías actuales se complementarán con estas últimas guías en los aspectos que conciernan a los medicamentos biosimilares. Además la guía de calidad (CPMP/BWP/3207/00) (EMEA, 2003) se reemplazará por la ICH Q5E $(\mathrm{ICH}, 2004)$ para los aspectos relacionados con los cambios en la calidad de los procesos de fabricación de productos biotecnológicos/ biológicos.

La tabla 1 recoge las directrices de comparabilidad establecidas para el desarrollo de medicamentos biológicos (EMEA, 2003; EMEA, 2007).

Tabla 1. Directrices de comparabilidad de medicamentos biotecnológicos (incluidos los biosimilares)

\begin{tabular}{|l|l|}
\hline EMEA/CPMP/BWP/3207/00 & $\begin{array}{l}\text { Directriz de comparabilidad de medicamentos que contienen } \\
\text { proteínas obtenidas por biotecnología como principio activo- } \\
\text { aspectos de calidad }\end{array}$ \\
\hline EMEA/CHMP/BMWP/101695/06 & $\begin{array}{l}\text { Directriz de comparabilidad de medicamentos obtenidos por } \\
\text { biotecnología tras un cambio en el proceso de fabricación - } \\
\text { aspectos no clínicos y clínicos }\end{array}$ \\
\hline
\end{tabular}

\section{GUÍAS APLICABLES A TODOS LOS MEDICAMENTOS BIOSIMILARES}

La tabla 2 recoge algunas de las guías existentes que son relevantes y deben tenerse en cuenta en la fase de desarrollo de un medicamento biosimilar y durante el estudio de comparabilidad para demostrar que el producto es similar a otro ya autorizado en la UE (EMEA, 1999; ICH, 1995; $\mathrm{ICH}, 1997$; ICH,1999). 
Tabla 2. Guías aplicables al desarrollo farmacéutico de medicamentos biotecnológicos (incluidos los biosimilares)

\begin{tabular}{|l|l|}
\hline \multirow{2}{*}{ EMEA/CPMP/BWP/328/99 } & $\begin{array}{l}\text { Desarrollo farmacéutico para productos biotecnológicos y biológicos } \\
\text { - Anexo a la Nota a la Guía de Desarrollo Farmacéutico. } \\
\text { CPMP/QWP/155/96 }\end{array}$ \\
\hline ICH Q5C & $\begin{array}{l}\text { Nota a la Guía de calidad de productos biotecnológicos: ensayos de } \\
\text { estabilidad de productos biotecnológicos/ biológicos. } \\
\text { CPMP/ICH/138/95-adoptada Dic.95 }\end{array}$ \\
\hline ICH Q6B & $\begin{array}{l}\text { Nota a la Guía de especificaciones: ensayos y criterios de } \\
\text { aceptación para productos biotecnológicos/ bicos. } \\
\text { CPMP/ICH/365/96-adoptada Marzo } 99\end{array}$ \\
\hline ICH S6 & $\begin{array}{l}\text { Nota a la evaluación de la seguridad preclínica de productos } \\
\text { obtenidos por biotecnología. CPMP/ICH/302/95 - adoptada Sept.97 }\end{array}$ \\
\hline
\end{tabular}

En la UE se han aprobado hasta la fecha un número reducido de medicamentos biosimilares, los cuales consisten en dos productos recombinantes de somatropina, cinco eritropoyetinas y cinco filgrastims (tabla 3).

Tabla 3. Biosimilares comercializados en Europa (Dic. 2009)

\begin{tabular}{|c|c|c|}
\hline Medicamento Biosimilar & $\begin{array}{c}\text { Denominación Genérica } \\
\text { Internacional }\end{array}$ & Medicamento de Referencia \\
\hline Omnitrope ${ }^{\circledR}$ & Somatropina & Genotropin ${ }^{\circledR}$ \\
\hline Valtropin ${ }^{\circledR}$ & Somatropina & Humatrope ${ }^{\circledR}$ \\
\hline Binocrit ${ }^{\circledR}$ & Eritropoyetina alfa & Eprex ${ }^{\circledR}$ \\
\hline Epoetin alfa hexal ${ }^{\circledR}$ & Eritropoyetina alfa & Eprex ${ }^{\circledR}$ \\
\hline Abseamed $®$ & Eritropoyetina alfa & Eprex ${ }^{\circledR}$ \\
\hline Silapo ${ }^{\circledR}$ & Eritropoyetina zeta & Eprex $®$ \\
\hline Retacrit ${ }^{\circledR}$ & Eritropoyetina zeta & Eprex $®$ \\
\hline Ratiograstim ${ }^{\circledR}$ & Filgrastim & Neupogen $®$ \\
\hline Biograstim ${ }^{\circledR}$ & Filgrastim & Neupogen ${ }^{\circledR}$ \\
\hline Tevagrastim ${ }^{\circledR}$ & Filgrastim & Neupogen ${ }^{\circledR}$ \\
\hline Zarzio $®$ & Filgrastim & Neupogen ${ }^{\circledR}$ \\
\hline Filgrastim Hexal ® & Filgrastim & Neupogen $®$ \\
\hline
\end{tabular}




\section{CONCLUSIONES}

Los fármacos obtenidos por biotecnología son una clase terapéutica con características diferenciales muy determinadas en relación con los fármacos convencionales que se obtienen por síntesis química o los que se extraen de productos naturales y por tanto los biosimilares no son a los medicamentos biológicos innovadores lo que los genéricos a los productos de síntesis química. Se demuestra que el simple término "genérico" no es aplicable y que los biosimilares no pueden ser regulados de la misma forma que los medicamentos genéricos.

No obstante, dado que los biosimilares se obtienen mediante procesos de fabricación cuya calidad se controla de forma similar a la de los productos biotecnológicos innovadores, es posible garantizar su eficacia y seguridad en ambos tipos de productos, las cuales se basan tanto en los estudios preclínicos y clínicos exigidos, como en la obligatoriedad para el laboratorio fabricante de implementar un plan de farmacovigilancia y gestión de riesgos tras su comercialización. Esto unido a la reducción que conlleva el uso de los biosimilares en los costes de los tratamientos permitirá a un mayor número de pacientes acceder a estos medicamentos.

\section{REFERENCIAS}

Covic, A. y M. Kuhlman, Biosimilars: recent developments. Int. Urol. Nephrol.: 39, 261-266 (2007).

Crommelin, D. y otros ocho autores, Pharmaceutical evaluation of biosimilars: important differences from generic low-molecular-weight pharmaceuticals. Eur J Hosp Pharm Sci.: 11, 11-17 (2005).

Directiva 2001/83/CE del Parlamento Europeo y del Consejo de 6 de noviembre de 2001, por la que se establece un código comunitario sobre medicamentos para uso humano (Diario Oficial, número $\mathrm{L}$ 311, de 28/11/2001; pp. 67-128). http://ec.europa.eu/ (2001).

EMEA/CPMP/BWP/328/99. Development Pharmaceutics for Biotechnological and Biological Products - Annex to Note for Guidance on Development Pharmaceutics. http://www.emea.europa.eu/ (1999).

EMEA/CPMP/BWP/3207/00. Guideline on comparability of medicinal products containing biotechnology-derived proteins as active substance: quality issues. http://www.emea.europa.eu/ (2003).

EMEA/CHMP/437/04.Guideline on Similar Biological Medicinal Products. http://www.emea.europa.eu/ (2005).

EMEA/74562/2006. Questions and Answers on biosimilar medicines (similar biological medicinal products) . http://www.emea.europa.eu/ (2007).

EMEA/CHMP/BMWP/101695/2006. Guideline on comparability of biotechnology-derived medicinal products after a change in the manufacturing process: non-clinical and clinical issues.

http://www.emea.europa.eu/ (2007).

HR 3590. Patient Protection and Affordable Care Act (USA). Disponible en:

http://democrats.senate.gov/reform/patient-protection-affordable-care-act-as-passed.pdf (2010).

ICH Q5C: Quality of Biotechnological Products: Stability Testing of Biotechnological/Biological Products. http://www.ich.org/ (1995).

ICH S6: Preclinical Safety Evaluation of Biotechnology-Derived Pharmaceuticals. Jul 1997 [citado 2207-2009]. http://www.ich.org/ (1997).

ICH Q6B. CPMP/ICH/365/96 - adoptada Marzo 99 (Topic Q6B. Step 4 Note for Guidance on Specifications: Test Procedures and Acceptance Criteria for Biotechnological/ Biological Products). http://www.ich.org/ (1999). 
ICH Q5E. ICH harmonised tripartite guideline. Comparability of biotechnological/biological products subject to changes in their manufacturing process. http://www.ich.org/ (2004).

Kresse, G., Biosimilars. Science, status, and strategic perspective. Eur. J. Pharm. Biopharm.: 72, 479-486 (2009).

Kuhlmann, M., D. Goldsmith y A. Covic, Biosimilars: current status. Int. J. Pharm. Med.: 21, 199-206 (2007).

Nowicki, M. Basic facts about biosimilars. Kidn. BI. Pres. Res.: 30, 267-72 (2007).

Roger, S. y A. Mikhail, Biosimilars: Opportunity or Cause for Concern?. J. Pharm. Pharmaceut. Sci.: 10, 405-410 (2007).

Schellekens, H. y E. Moors, Clinical comparability and European biosimilar regulations. Nature Biotechnol.: 28, 28-31 (2010).

Zopf, D. y G. Vergis, Glycosylation: a critical issue in protein development and manufacturing. Pharmaceutical Visions. Spring: 10-14 (2002).

Zuñiga L y B. Calvo, Regulating similar biological medicinal products. Trends Biotechnol.:27, 385-387 (2009).

Zuñiga, L. y B. Calvo, Global vision about the biological medicinal products: biosimilars. Curr. Pharm. Biotechnol.: 10,772-774 (2009).

Zuñiga, L. y B. Calvo, Biosimilars approval process. Regul. Toxicol. Pharmacol.: 56, 374-377 (2010).

Zuñiga, L. y B. Calvo, Biosimilars: pharmacovigilance and risk management. Pharmacoepidemiol. Drug Saf.: 19, 1-9 (2010). 
